\title{
Use of a cubic phase plate as solution for moderate astigmatism: preliminary study
}

\section{Uso de una lámina de fase cúbica como solución a astigmatismos moderados: estudio preliminar}

\author{
C. Almaguer ${ }^{1,2}$, J. Arines ${ }^{3, S^{*}}$ \\ 1. Departamento de Física Aplicada (área de Óptica), Facultade de Física, Campus Vida, Universidade de \\ Santiago de Compostela, España \\ 2. Departamento de Física, Centro Universitario de Ciencias Exactas e Ingenierías, Universidad de \\ Guadalajara, Mexico \\ 3. Departamento de Física Aplicada (área de Optometría), Facultade de Óptica e Optometría, Campus Vida, \\ Universidade de Santiago de Compostela, España.
}

(*) E-mail: justo.arines@usc.es S: SEDoPTICA member

Received: 07/10/2016 Accepted: 06/04/2017

DOI: $10.7149 /$ OPA.50.2.49014

\begin{abstract}
:
This work analyzes the use of a single cubic phasemask as a solution to moderate astigmatism of different orientations and magnitudes. The experimental results with artificial eye show that a cubic phasemask of the type $7.07 \mu \mathrm{m} \cdot\left(\mathrm{Z}_{3}{ }^{3}-\mathrm{Z}_{3}{ }^{-3}\right) @ 5 \mathrm{~mm}$ pupillary diameter, can be a solution to the astigmatism up to 3D with different orientations. The $\log$ MAR visual acuity obtained for all orientations is close to 0.05 for pupil sizes of $3 \mathrm{~mm}, 5 \mathrm{~mm}, 7 \mathrm{~mm}$. These results are a good starting point for the development of contact lenses or intraocular lenses that do not require stabilization systems increasing tolerance to errors in the correction of the astigmatism.

Key words:Astigmatism, neural transfer function, cubic phasemask, Intraocular Lenses, Contact Lenses, visual acuity.

\section{RESUMEN:}

En este trabajo se explora la posibilidad de utilizar una única lámina de fase cúbica como solución a astigmatismos moderados de distintas magnitudes y orientaciones. Los resultados experimentales obtenidos con ojo artificial muestran que una fase cúbica del tipo $7.07 \mu \mathrm{m} \cdot\left(\mathrm{Z}_{3}{ }^{3}-\mathrm{Z}_{3}{ }^{-3}\right) @ 5 \mathrm{~mm}$ diámetro pupilar, puede ser una solución al astigmatismo de hasta 3D con diferentes orientaciones. La agudeza visual logMAR obtenida para todas las orientaciones es superior a 0.05 logMar para tamaños de pupilas de $3 \mathrm{~mm}, 5 \mathrm{~mm}, 7 \mathrm{~mm}$. Estos resultados son un buen punto de partida para el desarrollo de lentes de contacto o lentes intraoculares con menos dependencia a la estabilización y que incrementen la tolerancia a errores en la determinación del eje y potencia del astigmatismo refractivo.
\end{abstract}

Palabras clave: Astigmatismo, función de transferencia neuronal, lámina de fase cúbica, lentes intraoculares, lentes de contacto, agudeza visual.

\section{REFERENCES AND LINKS / REFERENCIAS Y ENLACES}

[1] S. Vitale, L. Ellwein, M.F. Cotch, F.L. Ferris, R. Sperduto, "Prevalence of refractive error in the United States, 1999-2004,” Archives of ophthalmology 126(8),1111-1119 (2008).

https://doi.org/10.1001/archopht.126.8.1111 
[2] J.T. Holladay, J.R. Moran, G.M.Kezirian, "Analysis of aggregate surgically induced refractive change, prediction error, and intraocular astigmatism," J. Cataract Refract Surg. 27, 61-79 (2001). https://doi.org/10.1016/S0886-3350(00)00796-3

[3] E.R. Dowski , W.T. Cathey, "Extended depth of field through wave-front coding," Applied Optics 34(11), 1859-1866 (1995). https://doi.org/10.1364/AO.34.001859

[4] W. T. Cathey, E. R. Dowski, "New paradigm for imaging systems," Appl. Opt. 41, 6080-6092 (2002). https://doi.org/10.1364/A0.41.006080

[5] A. S. Burns, S. Marcos, A. E. Elsner, S.Bara, "Contrast improvement of confocal retinal imaging by use of phase-correcting plates," Opt. Lett. 27, 400-402 (2002) https://doi.org/10.1364/OL.27.000400

[6] J. Arines, R. O. Hernandez, S. Sinzinger, A. Grewe, and E. Acosta, "Wavefront-coding technique for inexpensive and robust retinal imaging," Opt. Lett. 39, 3986-3988 (2014) https://doi.org/10.1364/OL.39.003986

[7] W.T Cathey, Extended depth of field optics for human vision, US Patent 7025454B2, (2006). https://www.google.com/patents/US7025454

[8] P. Artal , "Towards 'super-vision': facts and fiction," Points de Vue 46, (2002). http://www.pointsdevue.com/magazine/points-de-vue-46

[9] Z. Zalevsky, Shai B. Yaish, O. Yehezkel, M. Belkin, "Thin spectacles for myopia, presbyopia and astigmatism insensitive vision," Opt. Express 15, 10790-10803 (2007) https://doi.org/10.1364/OE.15.010790

[10] J. Arines, C. Almaguer, E. Acosta,"Potential use of cubic phase masks for extending the range of clear vision in presbyopes: initial calculation and simulation studies," Ophthalmic Physiol. Opt 37(2), 141150 (2017). https://doi.org/10.1111/opo.12348

[11] L. K. Young, G. D. Love; H. E. Smithson "Accounting for the phase, spatial frequency and orientation demands of the task improves metrics based on the visual Strehl ratio", Vision Res. 90, 57-67 (2013) https://doi.org/10.1016/i.visres.2013.06.007

[12] P. Artal, L. Chen, S. Manzanera, D.R. Williams, "Temporal dependence of neural compensation for the eye's aberrations," Ophthalmol. Vis. Sci. 45(13), 1077 (2004).

[13] L. Chen, P. Artal, D. Gutierrez, D. R. Williams, "Neural compensation for the best aberration correction," Journal of Vision 7(10), 9 (2007). https://doi.org/10.1167/7.10.9

[14] A. B. Watson, A. J. Ahumada, Jr., " Modeling Acuity for Optotypes Varying in Complexity. Invest. Ophthalmol”. Vis. Sci. 51 (13), 5174 ( 2010). https://doi.org/10.1167/12.10.19

[15] A.B. Watson, A. J. Ahumada, Jr., "A standard model for foveal detection of spatial contrast," Journal of Vision 5 (9), 6 (2005). https://doi.org/10.1167/5.9.6

\section{Introducción}

Con frecuencia el ojo humano presenta errores refractivos que le impiden obtener una imagen óptima, siendo necesario el uso de soluciones ópticas como lentes oftálmicas (LO), lentes de contacto (LC), lentes intraoculares (LIOs) o cirugía refractiva (CR). Uno de estos errores es el astigmatismo, caracterizado por proporcionar una visión borrosa y distorsionada tanto en visión lejana como próxima debido principalmente a la diferencia de curvatura de los meridianos principales de la córnea (pudiendo contribuir también la geometría del cristalino y/o su descentramiento). Frecuentemente se encuentra asociado a otro defecto refractivo como miopía o hipermetropía. Se presenta en aproximadamente el $36 \%$ de la población con una magnitud mayor a 1D [1]. Las lentes utilizadas para su corrección deben tener la orientación adecuada, ya que la tolerancia al error de orientación en ellas disminuye conforme aumenta la magnitud del astigmatismo [2]. Así, mientras que para una persona con 1D de astigmatismo un error en el ángulo de $20^{\circ}$ puede ser perfectamente tolerado, para una persona con 3D, este mismo error angular equivale a un error refractivo adicional de 1D. Con el fin de evitar la rotación indeseada del elemento corrector se propone el uso de monturas no circulares en el caso de gafas, mientras que las LC presentan sistemas de estabilización dinámicos por parpadeo o prismas balastrados que aumentan el peso de la LC 
en su zona inferior contribuyendo a mantener su orientación. En el caso de LIOs se usan hápticos que permiten la fijación de la lente y evitan su rotación. Sin embargo tras la cirugía de implantación puede ocurrir que ésta se rote, incline, descentre o se introduzca un astigmatismo corneal asociado a las incisiones corneales. En cualquiera de estos casos el astigmatismo se modifica y disminuye el nivel de corrección esperado.

En el ámbito de la instrumentación óptica existe un gran número de propuestas para conseguir sistemas que proporcionen imágenes de buena calidad en ambientes variables que puedan introducir aberraciones. En 1995 E.R. Dowski y W.T. Cathey [3,4] propusieron una técnica novedosa denominada Wavefront Coding (WFC) con el fin de obtener un sistema óptico que proporcionara mayor calidad de imagen en presencia de aberraciones como desenfoque, astigmatismo, o coma. La técnica combina una etapa de codificación del frente de onda mediante una lámina de fase cúbica (LFC) que proporciona una respuesta de impulso del sistema invariante a la presencia de aberraciones, con una etapa de post-procesado que se encarga de decodificar la imagen, actualmente esta técnica se emplea en sistemas de reconocimiento de iris y de huella dactilar, y en cámaras de teléfonos móvil sin sistema de enfoque [5,6]. Posteriormente W.T. Cathey propuso la posibilidad de emplear una LFC en el campo de la óptica oftálmica para mejorar la visión de sujetos con aberraciones oculares [7], considerando que la decodificación de la imagen sería realizada por el cerebro [8]. Trabajos previos realizados por Zalevsky et al. [9] demostraron que la extensión de la profundidad de foco se puede usar para corregir presbicia, miopía, hipermetropía y astigmatismo. En este sentido, hemos publicado recientemente [10] un estudio sobre el uso potencial de láminas de fase cúbica como las empleadas en la técnica de WFC para mejorar la visión de sujetos présbitas. Con este tipo de soluciones fuimos capaces de obtener, con un ojo artificial, un nivel de agudeza visual superior a $0.1 \log$ MAR en un rango de visión entre 0.4 y $6 \mathrm{~m}$. En este trabajo mostramos así mismo que la métrica comúnmente usada de Visual Strehl VSOTF no es adecuada para evaluar la viabilidad de soluciones sin simetría de revolución, siendo más adecuadas otras métricas que enfatizan el peso de la fase de la función de transferencia óptica, como la propuesta por Young et al [11]. Además mostramos que la pérdida de contraste inducida por este tipo de soluciones es tolerable por el sistema visual gracias en parte al aumento de contraste que aporta la función de transferencia neuronal (NTF) y a la capacidad del cerebro de adaptarse al desenfoque y al contraste [12-13].

En este trabajo continuamos evaluando los usos potenciales de las soluciones ópticas basadas en fase cúbica. En este caso nos centraremos en la corrección del astigmatismo moderado, considerando que pueda ser utilizada en forma de lente de contacto o lente intraocular menos dependiente de la orientación y que incrementen la tolerancia a errores en la determinación del eje y potencia del astigmatismo.

\section{Materiales y Métodos}

Empezamos este apartado describiendo el montaje desarrollado para la valoración experimental de la propuesta. El sistema consistió en un ojo artificial y un conjunto de optotipos que se colocaron a diferentes distancias. El ojo artificial lo construimos con un diafragma variable ( $3 \mathrm{~mm}, 5 \mathrm{~mm} \mathrm{y} 7 \mathrm{~mm}$ ) y una lente plano-convexa de $25.4 \mathrm{~mm}$ de focal. Un objetivo de microscopio de $4 \mathrm{X}$, y apertura numérica 0.1 se acopló a una cámara modelo ORCA 285 Hamamatsu Photonics con tamaño de píxel de $8.1 \mu \mathrm{m}$. La distancia entre el objetivo de microscopio y el detector de la cámara que se estableció, proporciono una relación de aumento $3 \mathrm{X}$ entre el plano objeto e imagen del objetivo (coincidentes con el plano imagen del ojo artificial, y el detector de la cámara, respectivamente). De esta forma el tamaño efectivo de píxel considerando los aumentos del objetivo fue de $2.7 \mu \mathrm{m}$, tamaño comparable al de los fotorreceptores de la fóvea humana. Delante del ojo artificial colocamos una LFC de la forma $7.07 \mu \mathrm{m} \cdot\left(\mathrm{Z}_{3}{ }^{3}-\mathrm{Z}_{3}{ }^{-3}\right) @ 5 \mathrm{~mm}$ diámetro pupilar.

Para generar el astigmatismo utilizamos lentes esferocilíndricas-negativas (LEC) de magnitud $(0.75,1$, $1.50,1.75,2,2.50$ y 3 ) D. Para cada una de estas magnitudes se tomaron imágenes con tres orientaciones distintas del eje del astigmatismo $(0,30 \text { y } 45)^{\circ}$. En la figura 1 se muestra el arreglo experimental. 


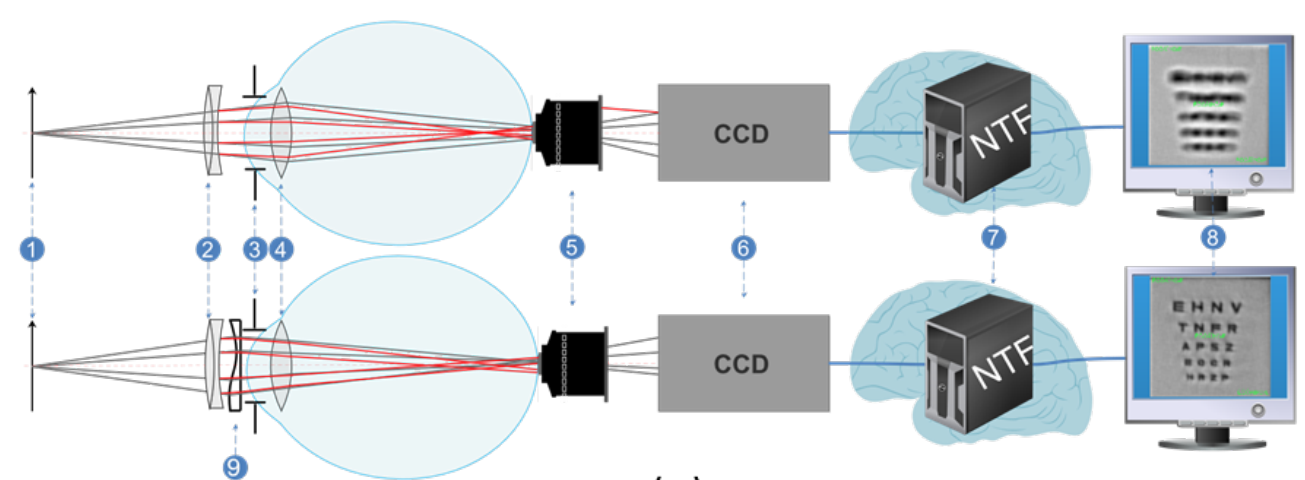

(a)

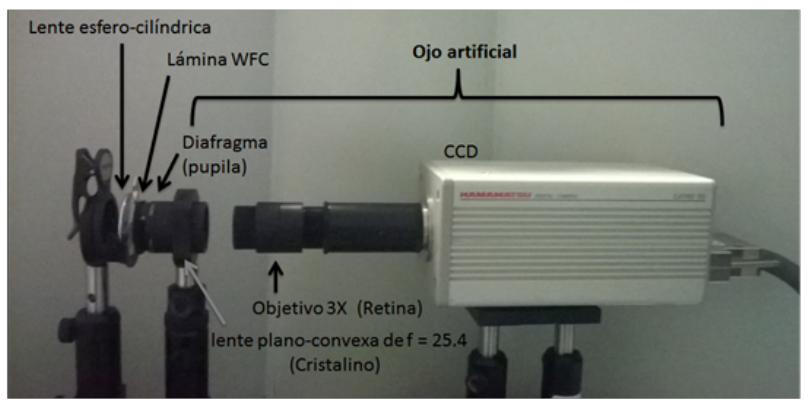

(b)

Fig. 1. a) Esquema del arreglo experimental: En la parte superior el ojo présbita desnudo y en la parte inferior ojo présbita con LFC. Los números indican los componentes:1) Optotipos, 2) LEC 3) Diafragma variable (Pupila), 4) lente plano-convexa de 25.4 mm, 5) Objetivo de microscopio, 6) CCD (retina), 7) Aplicación del filtro con la NTF (proceso neuronal), 8) Imágenes Obtenidas y 9) LFC; b)Montaje experimental.

La LFC fue realizada en polimetilmetacrilato (PMMA) mediante fresado de alta precisión [6]. En la figura 2 se muestra una imagen de la lámina de fase (fig. 2a), su mapa topográfico (fig. 2b) y la respuesta de impulso asociada (fig. 2c).

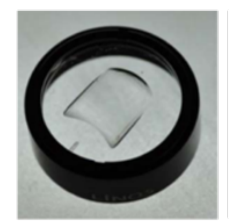

(a)

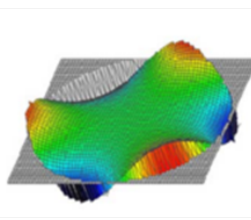

(b)

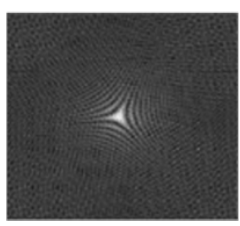

(c)

Fig. 2. LFC. a) Imagen de la LFC, b) Mapa de fase y c) PSF.

Con el fin de incluir la mejora de contraste asociado al procesado neuronal y simular parte del trabajo del cerebro, filtramos las imágenes registradas con la función de transferencia neural (NTF)[14]:

$$
\operatorname{NTF}\left(f, \theta ; f_{0}, f_{1}, a\right)=S_{E m G}\left(f ; f_{0}, f_{1}, a\right) \cdot \operatorname{OEF}(f, \theta)
$$

donde $\mathrm{S}_{\mathrm{EmG}}$ es el filtro de sensibilidad al contraste Gaussiano (ec.2) y OEF es el efecto oblicuo (ec. 3).

$$
S_{E m G}\left(f ; f_{0}, f_{1}, a\right)=\left[\exp \left(-\frac{f}{f_{o}}\right)-a \cdot \exp \left[-\left(\frac{f}{f_{1}}\right)^{2}\right]\right]
$$




$$
\begin{aligned}
& O E F(f, \theta)=\left[\exp \left(-\frac{f}{f_{o}}\right)-a \cdot \exp \left[-\left(\frac{f}{f_{1}}\right)^{2}\right]\right] .
\end{aligned}
$$

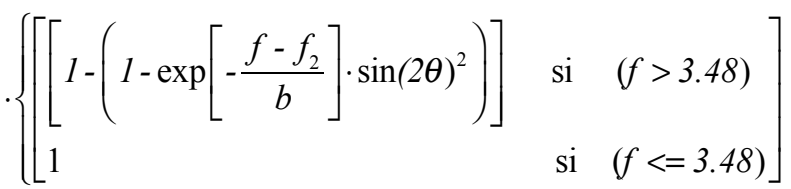

con valores: $\mathrm{f}_{0}=33.3573 \mathrm{f}_{1}=5.37916$ y a=0.923853 $\gamma=3.48$ ciclos $/$ grado $\mathrm{y} \lambda=13.57$ ciclos $/$ grado $[14,15]$.

La figura 3 se muestra la gráfica de la NTF utilizada (fig. 3a), pudiéndose observar que el efecto oblicuo (fig. 3a) reduce los valores umbrales de la función de sensibilidad al contraste a medida que la información relevante se aleja de los ejes vertical y horizontal.

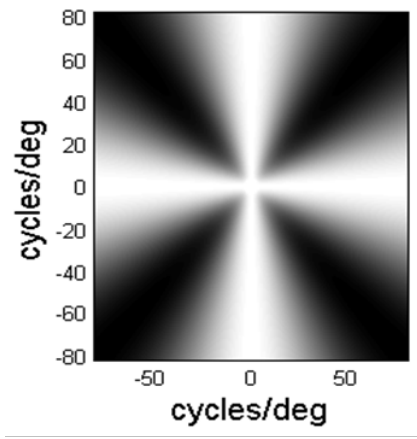

(a)

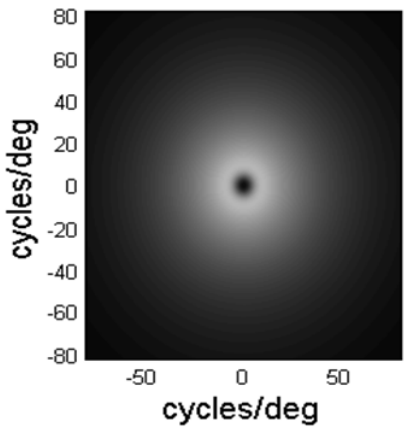

(b)

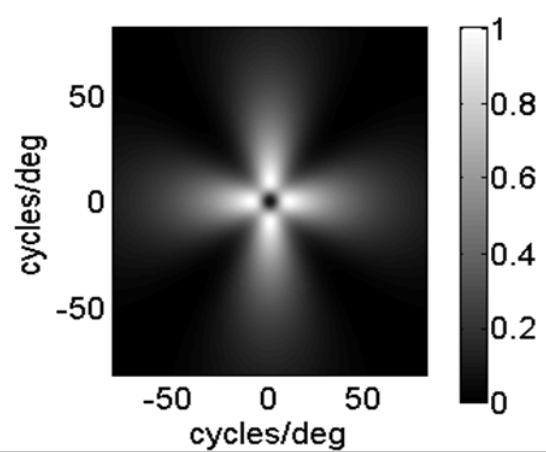

(c)

Fig. 3: a) OEF; b) $\mathrm{S}_{\mathrm{EmG}}$; c) NTF.

Como objeto se empleó una transparencia colocada a una vergencia objeto de $6 \mathrm{~m}$ respecto al ojo artificial, con un conjunto de filas con letras de distinto tamaño angular o agudeza visual (AV) de $(0.00,0.05,0.10$, 0.15 y 0.22 ) $\log M A R$, e iluminada con una fuente de luz halógena.

El protocolo de medidas fue el siguiente:

1) Ajustar la pupila a 5m; 2) Colocar lente LEC con el eje a 0 o; 3) enfocar la imagen moviendo el conjunto cámara-objetivo; 4) Registrar la imagen; 5) Repetir pasos 2 a 4 para orientaciones del eje del astigmatismo a $30^{\circ}$ y $45^{\circ}$; 6) Cambiar la LEC y repetir pasos 2 a 5; 7) Cambiar pupila y repetir pasos 2 a 6.

El protocolo descrito se empleó tanto con el ojo artificial desnudo (sin LFC) como tras colocar la LFC, en ambos conjuntos de imágenes se aplicó el filtro neuronal NTF. Tras obtener el conjunto de imágenes con y sin empleo de la LFC decidimos que la valoración de la calidad visual de las mismas y la estimación de la línea de agudeza visual máxima que se puede visualizar no la debíamos hacer nosotros, debido al sesgo que presentamos como conocedores de los optotipos y al probable proceso de adaptación al aspecto de las letras resultante de la visualización repetida de dichas imágenes. Por ello se proyectó una encuesta online. Las imágenes registradas y filtradas con la NTF fueron almacenadas en una plataforma de encuestas online para ser posteriormente evaluadas por un conjunto de 20 participantes, a los que se les pidió que indicaran cual era la línea de mayor agudeza visual en la que eran capaces de identificar al menos 3 letras.

En la encuesta se incluyeron únicamente los casos para (1, 2 y 3) D debido a que los casos intermedios $(0.75,1.50$ y 1.75$)$ D presentaron resultados similares, lo que permitió reducir el tamaño de la encuesta.

\section{Resultados}

Tras la toma de datos se obtuvieron dos conjuntos medidas (ojo desnudo (sin LFC) y ojo con LFC) de tres tamaños pupilares, tres orientaciones y siete magnitudes de astigmatismo, con un total de 126 imágenes. En la figura 4 se muestra un ejemplo de las distintas imágenes experimentales proporcionadas por el ojo artificial. 


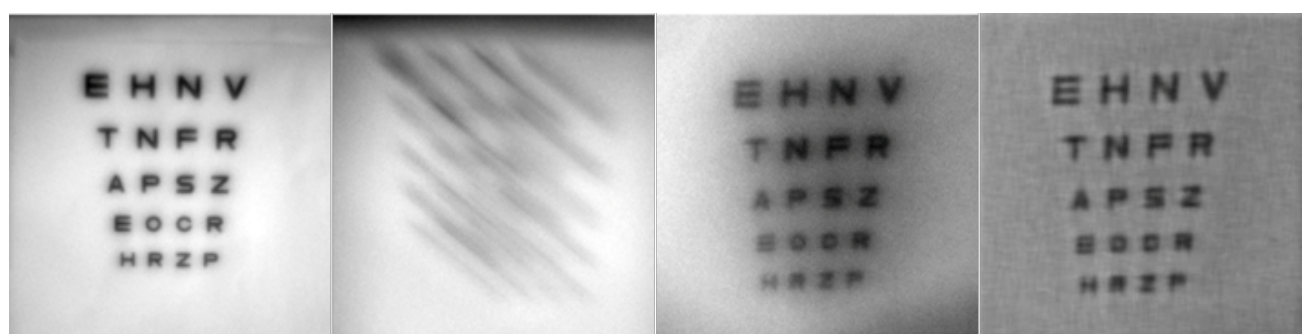

$\begin{array}{llll}\text { (a) } & \text { (b) } & \text { (c) } & \text { (d) }\end{array}$

Fig. 4. Imágenes capturadas por el ojo artificial: a) Sin aberraciones, b) con astigmatismo ( $3 \mathrm{D}$ a $45^{\circ}$ ), c) colocando la LFC al sistema de imagen y d) Imagen con LFC tras filtrado con la NTF.

En la figura 5 se presenta una muestra representativa de las imágenes obtenidas con el ojo desnudo (sin LFC) y con LFC para las magnitudes de astigmatismo $\left(0.75,1,2\right.$ y 3) D y ejes $\left(0,30\right.$ y 45) ${ }^{\circ}$. Ambos conjuntos de imágenes fueron filtrados con la NTF con el fin de incluir la mejora de contraste introducida por el sistema visual. Podemos observar como con una única lámina de fase cúbica se consigue obtener una calidad de imagen suficiente para un gran rango de potencias de astigmatismo, con una reducida dependencia respecto a la orientación del mismo. También se observa una disminución de contraste a medida que aumenta la potencia del astigmatismo. Cabe aclarar que la calidad de imagen que obtenemos con este elemento corrector es inferior a la que se obtiene compensando el astigmatismo con la lente esferocilíndrica adecuada (ver fig.4a como ejemplo de las imágenes que se obtienen compensando perfectamente el error refractivo). Por otra parte aunque existe una pérdida de contraste, la mejora en la calidad de imagen para cualquier magnitud de astigmatismo, eje y tamaño pupilar es notoria. Además podemos observar que en el caso sin LFC, al alcanzar las $2 \mathrm{D}$ de astigmatismo no es posible visualizar las letras, ni si quiera las de AV igual a $0.22 \log M A R$. 


\begin{tabular}{|c|c|c|c|c|c|c|c|}
\hline & & \multicolumn{6}{|c|}{ Pupila } \\
\hline \multicolumn{2}{|c|}{ Dioptría } & $3 \mathrm{~mm}$ & $5 \mathrm{~mm}$ & $7 \mathrm{~mm}$ & $3 \mathrm{~mm}$ & $5 \mathrm{~mm}$ & $7 \mathrm{~mm}$ \\
\hline \multirow{3}{*}{ 星 } & 8 & 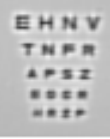 & 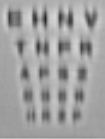 & $\begin{array}{c}\text { EHUv } \\
+ \text { Hen } \\
\text { yit: }\end{array}$ & $\begin{array}{l}\text { EHNV } \\
T N F E \\
A B S Z \\
\ldots E D\end{array}$ & $\begin{array}{l}\text { EHNV } \\
T N F= \\
A P E Z \\
\ldots O E\end{array}$ & 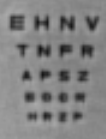 \\
\hline & $\stackrel{\wp}{\circ}$ & 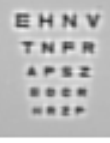 & 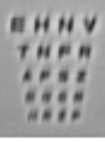 & 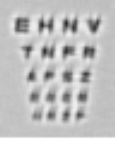 & 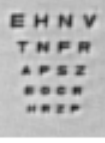 & $\begin{array}{l}\text { EHNV } \\
T N F n \\
A=S Z \\
\forall: B N\end{array}$ & 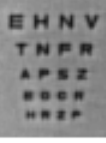 \\
\hline & if & 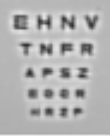 & 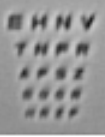 & 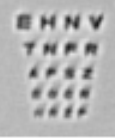 & 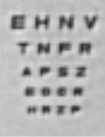 & 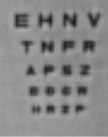 & $\begin{array}{l}E H N V \\
T N F R \\
A P S Z \\
: D E Z \\
W E Z\end{array}$ \\
\hline \multirow{3}{*}{ 旦 } & 8 & 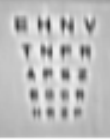 & 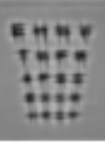 & Ex:? & 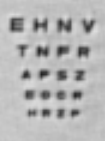 & 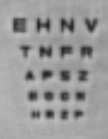 & 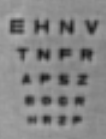 \\
\hline & હ) & 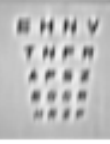 & 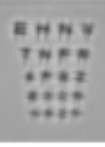 & 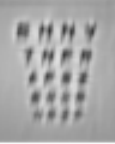 & 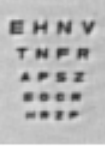 & 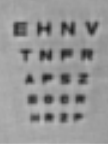 & $\begin{array}{l}E H N V \\
T N F R \\
A N=Z \\
\forall B E \\
H=E\end{array}$ \\
\hline & 祼 & 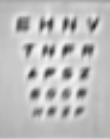 & 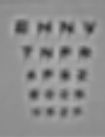 & 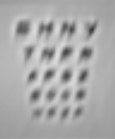 & 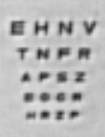 & 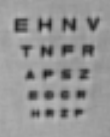 & 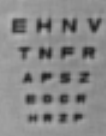 \\
\hline \multirow{3}{*}{ 穵 } & 8 & 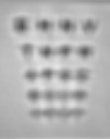 & rens & 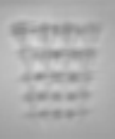 & 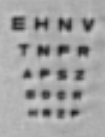 & 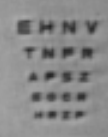 & 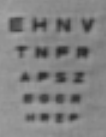 \\
\hline & $\stackrel{8}{8}$ & 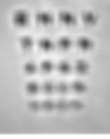 & 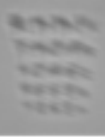 & 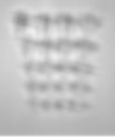 & $\begin{array}{l}\text { EHNV } \\
T M F R \\
\because E S Z \\
\because B E\end{array}$ & 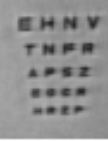 & 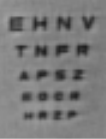 \\
\hline & if & 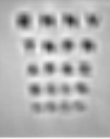 & $\frac{6 x}{4}$ & 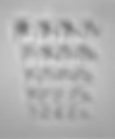 & 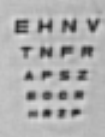 & 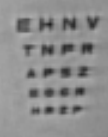 & 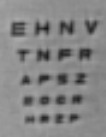 \\
\hline \multirow{3}{*}{ ค } & 잉 & netsen & nese & 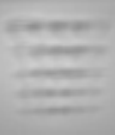 & $\begin{array}{l}E H N V \\
T N=n \\
\# S E \\
\ldots E V\end{array}$ & 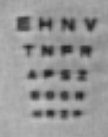 & 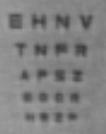 \\
\hline & $\stackrel{8}{\circ}$ & 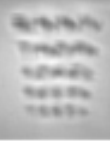 & & 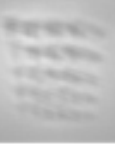 & 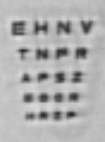 & 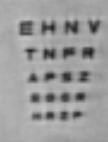 & 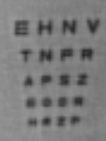 \\
\hline & is & 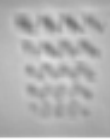 & & 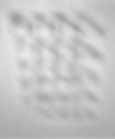 & 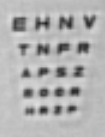 & 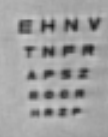 & 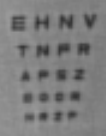 \\
\hline & & \multicolumn{3}{|c|}{ Sin LFC } & \multicolumn{3}{|c|}{ Con LFC } \\
\hline
\end{tabular}

Fig. 5: Imágenes de los optotipos obtenidas con el ojo artificial para astigmatismos de $(0.75,1,2,3) \mathrm{D}$, orientaciones $(30,45 \text { y } 60)^{\circ}$, y diámetros pupilares de $(3,5$ y 7$) \mathrm{mm}$. 
Finalmente en la figura 6 presentamos los resultados de la encuesta. En particular mostramos mediante un gráfico de barras el porcentaje de participantes que refirió alcanzar una determinada AV, para las distintas magnitudes de astigmatismo, orientación y diámetro pupilar. Como resultado a destacar vemos que cerca del 80\% de los encuestados manifestó ser capaz de identificar la línea de letras de AV 0.1 logMAR o superior, para cualquier diámetro pupilar y orientación de astigmatismo hasta 2D de magnitud.

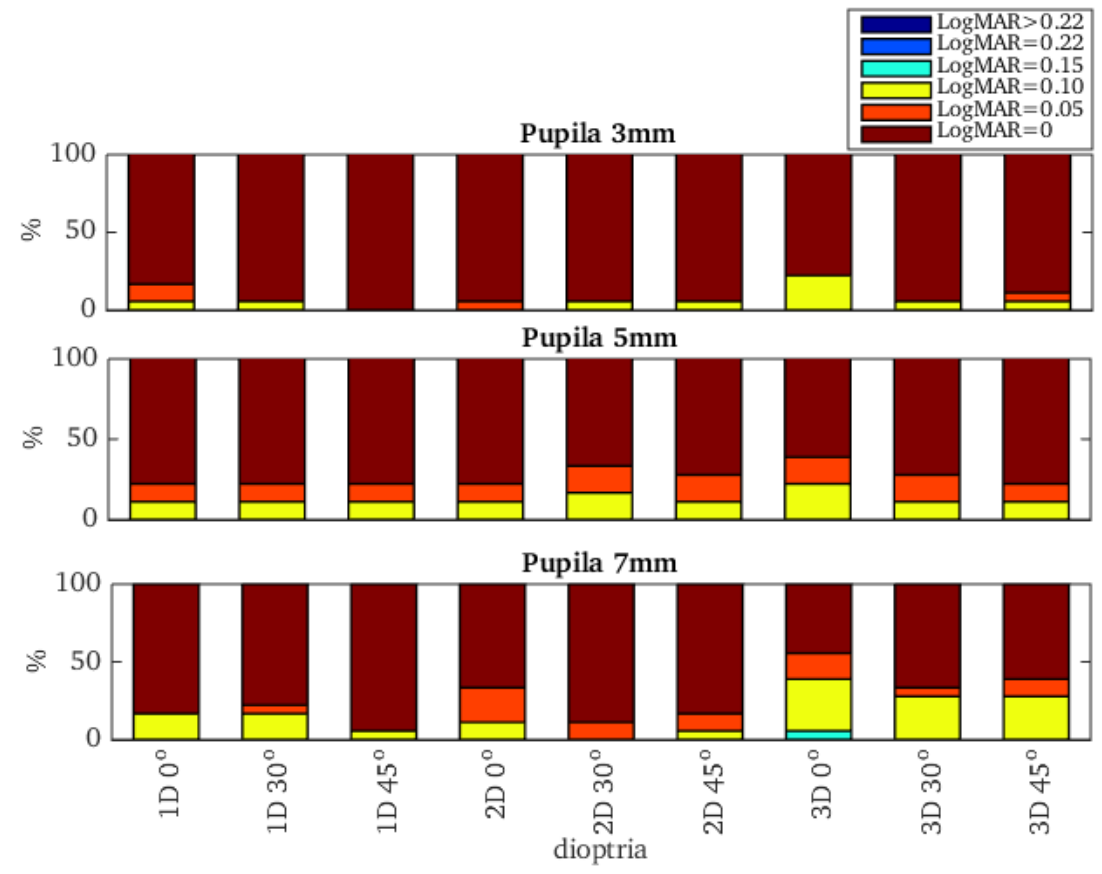

Fig. 6: Gráfico de barras con los resultados de las encuestas, donde se indica el porcentaje de encuestados que seleccionaron un mismo valor de logMAR para una distancia al optotipo de $6.0 \mathrm{~m}$ en función de la magnitud del astigmatismo y su orientación.

Frente a los trabajos previos realizados por Zalesvky et al. [9] que proponían el uso de una lámina de fase binaria que se colocarían sobre una lente oftálmica de forma que gracias a efectos difractivos se consiguiera extender la profundidad de foco y corregir el astigmatismo, en este trabajo abordamos la viabilidad del uso de láminas de fase cúbica para tal fin. Dicha propuesta fue lanzada inicialmente por Cathey [7], sin embargo no llego a concretar la demostración de la misma. Recientemente, en un trabajo previo [10] conseguimos mostrar la potencialidad de la propuesta realizada por Cathey en el ámbito de la corrección de la presbicia, comprobando que la codificación de la imagen introducida por la fase cúbica genera principalmente una disminución del contraste de las imágenes retinianas sin pérdida significativa de su contenido en frecuencias. Dicha codificación es parcialmente resuelta por la función de transferencia Neuronal (NTF), la cual presenta un aspecto similar a un filtro pasa banda apodizando las frecuencias bajas, mejorando de esta forma el contraste. En este trabajo continuamos explorando la potencialidad de la lámina de fase cúbica y en particular analizamos la posibilidad de emplearla para mejorar la visión de sujetos con astigmatismo. Los resultados expuestos muestran que con una única LFC somos capaces de obtener imágenes aceptables para cualquier orientación del astigmatismo y potencias hasta 3D. Este comportamiento se alcanza incluso para distintos tamaños pupilares (tanto $3 \mathrm{~mm}$ como $5 \mathrm{~mm} \mathrm{y} 7 \mathrm{~mm}$ ).

El desarrollo de lentes de contacto con estas cualidades permitiría la fabricación de elementos menos dependientes del sistema de estabilización. En el caso de las lentes intraoculares pensamos que la LFC podría ser útil para reducir los problemas asociados a astigmatismos quirúrgicos, desplazamiento o errores en el cálculo de la lente intraocular. La tolerancia al error no solo se refiere a la rotación indeseada de la lente sino que también a una mala elección de la potencia refractiva de la misma, de forma que se espera una mejora visual en aquellas situaciones en las que la determinación del error refractivo es complicada (como por ejemplo en casos de catarata muy avanzada).

Desde el punto de vista de tener un elemento pancorrector, el comportamiento de la LFC muestra que sería posible fabricar LC o LIO estándar de forma que con un único producto se pudiera satisfacer la demanda de corrección refractiva de un amplio sector de la población astigmática reduciendo de esta forma el coste de cada unidad y las necesidades de almacenamiento de los establecimientos comerciales. 


\section{Conclusiones}

En este trabajo hemos mostrado la posibilidad de usar láminas de fase cúbica(como los usados en la técnica de Wavefront Coding) para la corrección de astigmatismos refractivos de diversa magnitud y orientación. En particular mostramos que utilizando una lámina de fase $7.07 \mu \mathrm{m} \cdot\left(\mathrm{Z}_{3}{ }^{3}-\mathrm{Z}_{3}{ }^{-3}\right)$ se consigue obtener imágenes de calidad visual aceptable en presencia de astigmatismos de entre 1 y 3 dioptrías y distintas orientaciones $(0,30 \text { y } 45)^{\circ}$. Consideramos que estos resultados son un buen punto de partida para motivar la realización de pruebas con ojos reales así como otros estudios en esta línea.

\section{Agradecimientos}

Este trabajo ha sido financiado por el Ministerio de Economía y Competitividad recursos FIS2013-46188P y FIS2016-77319-C2-1-R. 